\title{
The Simplicial Ricci Tensor
}

\author{
Paul M. Alsing ${ }^{1}$, Jonathan R. McDonald ${ }^{1,2}$ \& Warner A. \\ Miller ${ }^{3}$ \\ ${ }^{1}$ Information Directorate, Air Force Research Laboratory, Rome, New York 13441 \\ ${ }^{2}$ Insitut für Angewandte Mathematik, Friedrich-Schiller-Universität-Jena, 07743 \\ Jena, Germany \\ ${ }^{3}$ Department of Physics, Florida Atlantic University, Boca Raton, FL 33431 \\ E-mail: jonathan.mcdonald.ctr@rl.af.mil
}

\begin{abstract}
The Ricci tensor (Ric) is fundamental to Einstein's geometric theory of gravitation. The 3-dimensional Ric of a spacelike surface vanishes at the moment of time symmetry for vacuum spacetimes. The 4-dimensional Ric is the Einstein tensor for such spacetimes. More recently the Ric was used by Hamilton to define a non-linear, diffusive Ricci flow (RF) that was fundamental to Perelman's proof of the Poincarè conjecture. Analytic applications of RF can be found in many fields including general relativity and mathematics. Numerically it has been applied broadly to communication networks, medical physics, computer design and more. In this paper, we use Regge calculus (RC) to provide the first geometric discretization of the Ric. This result is fundamental for higher-dimensional generalizations of discrete RF. We construct this tensor on both the simplicial lattice and its dual and prove their equivalence. We show that the Ric is an edge-based weighted average of deficit divided by an edge-based weighted average of dual area - an expression similar to the vertex-based weighted average of the scalar curvature reported recently. We use this Ric in a third and independent geometric derivation of the RC Einstein tensor in arbitrary dimension.
\end{abstract}




\section{Introduction}

The Ricci curvature tensor (Ric) governs the dynamics of geometry in vacuum general relativity. It also has been pivotal in the mathematical classification of manifolds. It can therefore have a profound impact on our understanding of geometry and deepen our insights into classical and quantum gravity. Hamilton used the Ric to define a diffusive curvature flow that is referred to as Ricci flow (RF) [1];

$$
\left(\begin{array}{c}
\text { Rate of change } \\
\text { of the metric }
\end{array}\right)=-2 \text { Ric. }
$$

This was instrumental in Perelman's proof of Poincaré's conjecture [2, 3, 4]. In addition

to its mathematical applications, RF has been applied to a broad range of problems ranging from medical physics to network routing, and from face recognition to general relativity and cosmology. Many of the applications of RF are for discrete, unstructured meshes. Regge calculus (RC) provides a natural discrete description of Einstein's geometric theory of gravitation [5]. Here we apply $\mathrm{RC}$ to define the Ric in $\mathrm{RC}$ for arbitrary dimensions, so that RF can be extended to higher dimensions.

Evolutions of the Ric have found recent applications in the physics of spacetime. $\mathrm{RF}$ is expected to be an important tool for the study of generic black hole solutions of spacetime. For example, RF provides a means for a better understanding of quasilocal mass in non-trivial asymptotically flat spacetimes [6]. Moreover, it may be useful for a mathematically rigourous prescription for black hole boundary conditions in the numerical relativity community [7]. Similary, RF has also been applied to black-hole physics as a means for determining the Bekenstein-Hawking entropy [8, 9]. In cosmology, there has been increased interest in RF as means for understanding the averaging problem in $\Lambda$ CDM cosmological models [10, 11].

Numerical methods using RF techniques require discrete representations of the Ric and its corresponding evolution equation. Current RF techniques in computational geometry on complex topologies focus on 2-dimensional representations of higherdimensional data [12, 13]. Meanwhile, recent numerical simulations of relativistic models examined RF on higher dimensional manifolds with lower complexity topologies [14, 15, 16, 17]. Geometric discretizations of the Ric are needed for numerical simulation of RF on higher dimensional manifolds with arbitrary topology. RC is a natural setting for investigating the Ric and RF due to its piecewise-flat, coordinate-free construction which naturally captures the Riemannian curvature on each codimension 2 hinge, $h$, of the simplicial lattice. Here we use this RC Riemann curvature to derive a simplicial representation of the Ric. This one-form expression is valid in arbitrary dimension. We start by reviewing some of the principles related to representation of differential forms in $\mathrm{RC}$ and the notation used in this article in Section 2. In Section 3 we develop simplicial Ric on edges of the simplicial and dual lattices. In Section 4 we use our expression of the simplicial Ric to provide a third and independent geometric derivation of the RC Einstein tensor in arbitrary dimension. In particular, we utilize the simplicial Ric and scalar curvature to explicity construct the Einstein tensor as the trace-reversed Ric. 


\section{Dual Lattices and Discrete Differential Forms}

Geometric discretizations [12, 13, 18, 19, 20, 21] are generally characterized by association of tensors with lattice elements of a discrete manifold. Tensors decomposed into the space of values and tangent space components become weighted distributions over the skeleton of the discrete manifold and obtain their geometric properties from the skeleton itself. Differential quantities in the lattice are formulated such that point-

wise evaluation gives way to averaged evaluation over an integral domain. Tensors thus become integrated measures on the discrete manifold and their associated scalar weights may be intepreted as densities assigned to a lattice element. This integrated representation of tensors over lattice elements is a form of discrete exterior calculus or discrete differential forms (DDF) in which one explicitly discretizes the tangent space values of a differential form.

The simplicial lattice in RC provides one set of differential forms onto which a tensor may be projected. The simplicial $d$-volumes of a $d$-dimensional manifold provide an anchor - the tangent space- for the differential forms. However, to incorporate dual forms we require a lattice structure obtained by some duality relation with the simplicial skeleton, i.e. the dual lattice. We will often use the more generic phrasing of dual lattice to refer to the circumcentric dual lattice. The circumcentric dual lattice is the unique lattice defined by connecting the circumcenter of a $d$-simplex to the circumcenters of each neighboring $d$-simplex. This lattice is of special interest since it creates a pairwise orthogonality between elements of the dual lattice, i.e. for each $k$-element of the simplicial lattice there exists a $(d-k)$-element in the circumcentric dual. Moreover, if we constrain the simplicial lattice to be a Delaunay lattice [22], then the circumcentric dual is identified as a Voronoi lattice. In this particular case, the $d$-dimensional Voronoi cells are uniquely determined by the set of all points closest to a given simplicial vertex than to any other simplicial vertex. Likewise, a general $(d-k)$-Voronoi element is the set of all points in the codimension- $k$ hyperplane closest to a $k$-simplex than to any other $k$-simplex in the simplicial lattice. Thus, a $d$-volume constructed from the simplicial element and its Voronoi dual has a natural interpretation as the local, compact integral measures on the simplicial lattice. (See Appendix A for more details.)

Some of the notation used in this article will denote elements of the simplicial (dual) lattice, volumes in the lattices, or measures of curvature. In particular, we will distinguish between the simplicial and dual lattices using Latin and Greek lettering. The Latin letters $v, \ell$, and $t$ will label simplicial elements of dimension 0,1 , and 2, respectively. Arbitrary $k$-simplexes are labeled by $s^{(k)}$. Meanwhile, the elements of the dual lattice are labeled by the Greek letter counterparts $\nu, \lambda, \tau$, and $\sigma^{(k)}$. We will also be using the notation $\Delta V_{a}$ to denote the $d$-volume associated with the element $a$. For an edge $\ell$ of the simplicial lattice on a 3-dimensional lattice, the label $\Delta V_{\ell}$ represents a 3 -volume associated with $\ell$. The label $\Delta V_{a b}$ denotes the $d$-volume associated with $a$ and restricted to the element $b$. This restriction can be formulated as taking the intersection of the individual $d$-volumes from $a$ and $b$. Extending this notation to 
arbitrary restrictions, we can write $\Delta V_{a_{1} a_{2} \cdots a_{k}}$ as the restriction of the volume $\Delta V_{a_{1}}$ to all of the elements $a_{2}, \ldots, a_{k}$. Indeed, one can convince oneself of this notation by considering the case of the simplicial manifold restricted to a given element of either lattice. In this case, the entire manifold contains the $d$-volume of every lattice element, so $\Delta V_{a}$ can be seen to be the restriction of the simplicial manifold to the element $a$.

These notations, and others, are summarized below:

$$
\begin{aligned}
\nu & - \text { Dual vertex } \\
\lambda & - \text { Dual edge } \\
\tau, h^{*} & - \text { Dual polygon } \\
\sigma^{(k)} & - \text { Dual polytope of dimension } k \\
v & - \text { Simplicial vertex } \\
\ell & - \text { Simplicial edge } \\
t & - \text { Triangle on simplicial skeleton } \\
s^{(k)} & - \text {-simplex } \\
h & - \text { Simplicial hinge } \\
S t(a) & - \text { Star of a lattice element } a \text {, i.e. } \bigcup_{s^{(k)} \supset a} s^{(k)} \text { for the simplicial lattice } \\
A_{h},|h| & - \text { Volume of } h \\
A_{h}^{*},\left|h^{*}\right| & - \text { Area of } h^{*} \\
\left|s^{(k)}\right|\left(\left|\sigma^{(k)}\right|\right) & - \text { volume of } s^{(k)}\left(\sigma^{(k)}\right) \\
\theta_{h \ell} & - \text { Angle opposite of edge } \ell \text { on a hinge } h \text { in } 4 \text { dimensions } \\
\epsilon_{h} & - \text { deficit angle associated with a hinge } \\
R_{h} & - \text { Riemann Tensor projected on a hinge } \\
R_{\lambda} & - \text { Ric projected on a dual edge, } \lambda \\
R_{\ell} & - \text { Ric projected on a simplicial edge, } \ell \\
R_{\nu} & - \text { Ricci scalar at a dual vertex, } \nu \\
R_{v} & - \text { Ricci scalar at a simplicial vertex, } v \\
A_{h \ell} & - \text { Volume of hinge restricted to } \ell \\
A_{h \lambda}^{*} & - \text { Area of dual to a hinge restricted to } \lambda \\
|a|_{b} & - \text { Volume of } a \text { restricted to } b, \text { i.e. the norm of } a \bigcap b \\
|a|_{b_{1} \ldots b_{m}} & - \text { Volume of } a \text { restricted to all } b_{i}, \text { i.e. the norm of } a \bigcap b_{1} \bigcap \cdots \cap b_{m} \\
\Delta V_{a} & -d \text {-volume associated with the element (either dual or simplicial) } a . \\
\Delta V_{a b} & -d \text {-volume of } a \text { restricted to } b \\
\left\langle\alpha^{(k)}, s^{(k)}\right\rangle & - \text { Local projection or metric inner-product of two } k \text {-forms, } \\
\left(\alpha^{(k)}, \beta^{(k)}\right) & - \text { Standard } L_{2} \text { inner-product on two simplicial }(\text { dual }) k \text {-forms } \\
\hline\left\langle C_{a}\right\rangle_{b} & - \text { Volume-weighted average of the } C_{a} \text { 's hinging on the element } b, \frac{\sum_{a: b \in a} C_{a} \Delta V_{a b}}{\sum_{a: b \in a} a a_{a b}} \\
\left\langle C_{a}\right\rangle_{b} & - \text { Area-weighted average of the } C_{a} \text { 's hinging on the element } b, a \frac{\sum_{a: b a a} C_{a} A_{a b}}{\sum_{a: b \in a} A_{a b}} \\
\left.\bar{C}_{a}\right|_{b} & - \text { Arithmetic mean of the } C_{a} \text { 's hinging on } b \\
&
\end{aligned}
$$




\section{Discretizing the Ricci Tensor}

Here we construct a geometric representation of the Ric on a piecewise-flat simplicial geometry. The geometric discretization we use is based on discrete differential forms (DDF) in which the (dual) simplicial lattice is used as the (co-)chain complex for embedding continuum forms in the discrete manifold. It has been found that such discretizations preserve the geometric properties of the tensors and can be useful for solving differential equations for tensor fields on geometries with complex topology [20, 23, 21].

Piecewise-flat geometries are characterized by curvature distributions concentrated at each codimension 2 hinge, $h$, on the simplicial manifold, $\mathcal{S}$. The curvature on a given hinge $h$ is a conical singularity with deficit angle $\epsilon_{h}$. We have shown that standard RC is consistent with distributing this curvature evenly over the polyhedron, $h^{*}$, (with area $A_{h}^{*}$ ) dual to hinge $h$. It admits a natural interpretation as the sole independent component of the Riemann curvature tensor in the $d$-volume associated with the hinge [24]. From this local representation of curvature distributed over a hinge one can explicitly and

geometrically define the Einstein tensor in 4-dimensions [25] and a vertex-based scalar curvature [26]. The Einstein tensor encodes the geometrodynamics of General Relativity through the Einstein equations. The scalar curvature provides a point-wise average of curvature that an observer can set out to measure. However, these curvature measures are insufficient to examine geometric flows where the Ric plays the predominent role. When discretizing evolution processes that can be reformulated as an evolution of the Ric itself, e.g. RF, we seek to first represent the Ric directly in the geometry, then develop the evolution equations for the new representation. We provide two equivalent derivations of the Ric. First, we start with the continuum construction and apply it directly to discrete curvature forms. Second, we derive an equivalent expression directly from the action principle of RC.

\subsection{Derivation of the Ric from the Continuum using Discrete Curvature Forms}

In the continuum the Ric is defined as the first contraction of the Riemann curvature tensor;

$$
R_{b}^{a}=R_{b c}^{a c} .
$$

As a bivector-valued two-form the curvature tensor takes in a bivector for the loop of parallel transport and outputs a bivector characterizing the change in a vector transported around the loop;

$$
\mathbf{R}=\frac{1}{4} e_{a} \wedge e_{b} R_{c d}^{a b} d x^{c} \wedge d x^{d}
$$

where $\left\{e_{a}\right\}$ are the basis tangent vectors dual to the basis one-forms $\left\{d x^{c}\right\}$.

In $\mathrm{RC}$, curvature is given exclusively by the sectional curvature, $K$, associated with a codimension 2 hinge. On a hinge, the sectional curvature is given by

$$
K=\frac{\epsilon_{h}}{A_{h}^{*}}
$$




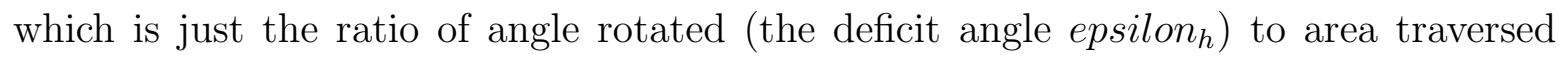
$\left(A_{h}^{*}\right)$ by the loop of parallel transport. The sectional curvature is the double projection of the Riemann tensor onto a given plane [27];

$$
K=\mathbf{R}\left(e_{a}, e_{b}, e_{a}, e_{b}\right)
$$

where $e_{a}$ and $e_{b}$ are an orthonormal basis for the plane. Hence the Riemann curvature tensor on a hinge is proportional to the sectional curvature of the polygonal dual, $h^{*}$, to the hinge;

$$
\mathcal{R}_{h}=R\left(h_{a b}^{*}, h^{*, a b}\right)=d(d-1) \frac{\epsilon_{h}}{A_{h}^{*}} .
$$

For this reason, one can generally denote the Riemann tensor for a hinge as $R^{h^{*}} h^{*}$. We will, in general, only keep track of the two-form components and write $R_{h^{*}}=R_{h}$, where the equality is a result of the duality. Taking the trace of the Riemann tensor requires summation over the curvature associated with loops spanned, in part, by a given oneform $e^{b}$. This summation of loops hinging on a given one-form reduces the curvature two-form to a one-form doubly-projected on $e^{b}$.

$\mathrm{RC}$ is at its heart a weak variational formulation of General Relativity. This is easily seen since the geometric content of $\mathrm{RC}$ is encoded not through pointwise defined tensors, but tensors distributed over elements of the lattice. Indeed, the Regge equations are integral equations and given by the Einstein tensor integrated over the associated 4volume. Hence, we evaluate the discrete Ric as an integrated quantity on the simplicial manifold. Locally, the Ric becomes a one-form projected on the dual edges of the lattice and integrated over the $d$-dimensional domain, $\Delta V_{\lambda}$, associated with the dual edge, $\lambda$. To take the trace of the Riemann tensor, one must sum over the independent directions orthogonal to a dual-edge $\lambda$. In general, one will sum over all independent two-forms $\lambda \wedge e^{a}$. However, when $e^{a}$ lies in the plane of a hinge $h$, there is no curvature associated with such a loop of parallel transport. Therefore, the Ricci one-form on $\lambda$ is dependent only on the the polyhedral 2 -faces, $h^{*}$, hinging on a given dual edge, $\lambda$;

$$
R_{\lambda} \Delta V_{\lambda}=\sum_{h^{*}: \lambda \in h^{*}} R_{h^{*}} \Delta V_{h^{*} \lambda}
$$

We have introduced the volume $\Delta V_{h^{*} \lambda}$ (Figure 1) which is a restriction of the $d$-volume for $h^{*}$ to the dual edge $\lambda$-the intersection of the $d$-volumes associated with $h^{*}$ and $\lambda$. This is the discrete equivalent of decomposing a domain and integrating over distinct representations on the subdomains;

$$
\int_{\Omega} \alpha=\sum_{i} \int_{\Omega_{i}} \alpha^{\prime}\left(\Omega_{i}\right) .
$$

Here the $\Omega_{i}$ form a non-overlapping domain decomposition of $\Omega$. Using the VoronoiDelaunay orthogonal decomposition of volumes and the $\mathrm{RC}$ definition of curvature on a hinge, $R_{h}=d(d-1) \frac{\epsilon_{h}}{A_{h}^{*}}$, we obtain an explicit expression for the integrated Ricci one-form on a dual edge;

$$
R_{\lambda} \Delta V_{\lambda}=\sum_{h^{*}: \lambda \in h^{*}} d(d-1) \frac{\epsilon_{h}}{A_{h}^{*}} \frac{1}{\left(\begin{array}{l}
d \\
2
\end{array}\right)} A_{h} A_{h \lambda}^{*}
$$




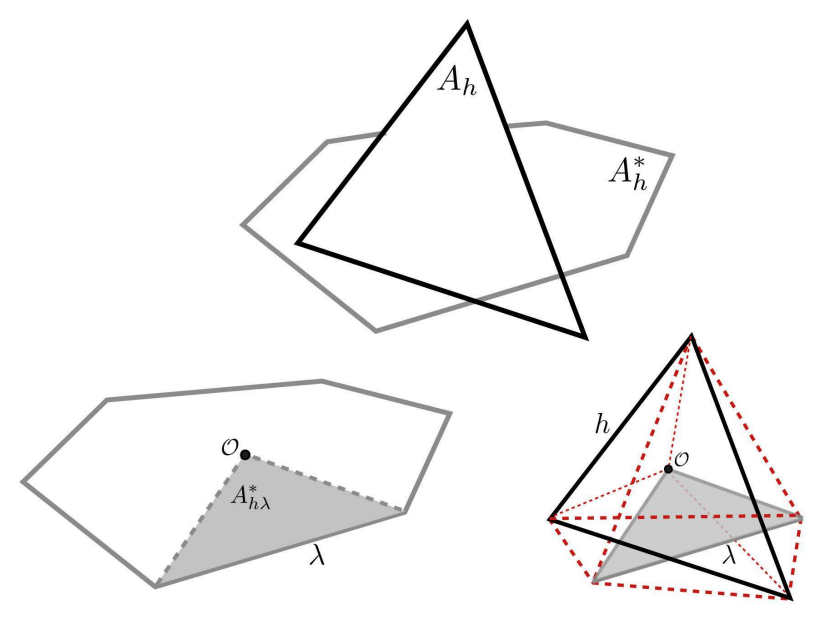

Figure 1. Restricting the Hinge Volume to a Dual Edge: Here we explicitly show the decomposition of the d-volume of a hinge $h($ in $d=4)$ and its restriction to a dual edge $\lambda$. (Top) Here we show the orthogonal decomposition of the $d$-volume into the area of a hinge, $A_{h}$, and the area of the dual polygon to a hinge, $A_{h}^{*}$. Struts (notshown) connecting each vertex of $h^{*}$ to each vertex of $h$ complete the boundary of the domain spanned by $h$ and $h^{*}$. (Bottom-left) We focus attention on the dual polygon $h^{*}$ and have shown (shaded) the restriction to the dual edge $\lambda$. This restricted area is the 2-simplex constructed from the endpoints of $\lambda$ and the circumcenter of the hinge $h$. (Bottom-right) By connecting the vertexes of the restricted area of $h^{*}, A_{h \lambda}^{*}$, to each of the vertexes of the hinge $h$, we obtain a new $d$-volume, $\Delta V_{h^{*} \lambda}=\Delta V_{h \lambda}$. The thick red (dashed) lines are struts connecting vertexes on the boundary of $\Delta V_{h^{*} \lambda}$ The struts connecting the circumcenter $\mathcal{O}$ of $h$ to the vertexes of $h$ (thin dashed, red) do not contribute the boundary of $\Delta V_{h \lambda}$ and can be routinely dropped from the construction.

$$
=\sum_{h^{*}: \lambda \in h^{*}} 2 \epsilon_{h} A_{h} \frac{A_{h \lambda}^{*}}{A_{h}^{*}} .
$$

We have decomposed the restricted $d$-volume, (see Appendix A), into the Voronoi and Delaunay components and restricted the Voronoi area, $A_{h}^{*}$, to the dual edge, $\lambda$, denoted as $A_{h \lambda}^{*}$. This restricted area is the set of all points in $A_{h}^{*}$ closer to $\lambda$ than to any other dual edge $\lambda^{\prime}$ in the skeleton of $h^{*}$. Dividing by the intergal domain, we obtain

$$
R_{\lambda}=\frac{\sum_{h^{*}: \lambda \in h^{*}} d(d-1) \frac{\epsilon_{h}}{A_{h}^{*}} A_{h \lambda}^{*} A_{h}}{\sum_{h^{*}: \lambda \in h^{*}} A_{h \lambda}^{*} A_{h}}=\frac{\sum_{h^{*}: \lambda \in h^{*}} R_{h} \Delta V_{h \lambda}}{\sum_{h^{*}: \lambda \in h^{*}} \Delta V_{h \lambda}} .
$$

Defining a volume-weighted average as

$$
{\overline{\left\langle C_{a}\right\rangle_{b}}}_{b}=\frac{\sum_{a: b \in a} C_{a} \Delta V_{a b}}{\sum_{a: b \in a} \Delta V_{a b}}
$$

the Ricci one-form in the dual lattice becomes

$$
R_{\lambda}={\overline{\left\langle R_{h}\right\rangle_{\lambda}}}
$$

This is an explicit expression of the Ric in the dual lattice as a weighted average of curvatures meeting on the dual lattice one-form $\lambda$. 
In RC, it is customary for measures of curvature to be associated with elements in the simplicial lattice. This more readily allows for evolution equations in terms of the degrees of freedom, the edge lengths of the simplexes $\{\ell\}$. For applications of the Ric, such as for RF, this is particularly important since a straightforward weak evolution equation for the edge lengths (synonomous with the components of metric) will require an integration of the Ric over the $d$-volume associated with an edge, i.e. the integrated Ricci one-form at a given $\ell$. We thus seek to re-express the Ricci one-form on the simplicial skeleton. Taking the dual of the above expression gives us a Ricci three-form on the simplicial lattice. However, it is beneficial to write an explicit expression for the Ricci one-form on simplicial edges. We can transform the above expression into a edge-based expression in the simplicial skeleton via a lowering (raising) operator which transforms $r$-forms in the dual (simplicial) lattice to $r$-forms in the simplicial (dual) lattice (see Appendix B). We first rewrite the association of the Ric on a dual edge by restricting the domain to that closest to a simplicial edge, $\ell$. This is the result of the projection of the dual edge Ric onto the domain of the edge, $\ell$;

$$
\begin{aligned}
R_{\lambda} \Delta V_{\lambda \ell} & =R_{\lambda} \Delta V_{\lambda} \frac{\Delta V_{\lambda \ell}}{\Delta V_{\lambda}} \\
& =\sum_{h^{*}: \lambda \in h^{*}} R_{h^{*}} \Delta V_{h^{*} \lambda \ell} .
\end{aligned}
$$

For $d>2$ this newly projected volume can be decomposed as before, except now we must restrict the hinge area to that which is closest to $\ell$. Suitably rearranging the terms in the sums gives

$$
\begin{aligned}
R_{\ell} \Delta V_{\ell} & =\sum_{\lambda \in \ell^{*}} R_{\lambda} \Delta V_{\lambda \ell} \\
& =\sum_{\lambda \in \ell^{*}} \sum_{h^{*}: \lambda \in h^{*}} 2 \epsilon_{h} A_{h \ell} \frac{A_{h \lambda}^{*}}{A_{h}^{*}} \quad(\text { for } d>2) \\
& =\sum_{h: \ell \in h} \frac{2 \epsilon_{h} A_{h \ell}}{A_{h}^{*}} \sum_{\lambda \in h^{*}} A_{h \lambda}^{*} \\
& =2\left\langle\epsilon_{h}\right\rangle_{\ell} A_{h}
\end{aligned}
$$

where we have defined the edge-based area-weighted average as

$$
\left\langle C_{h}\right\rangle_{\ell}=\frac{\sum_{h: \ell \in h} C_{h} A_{h \ell}}{\sum_{h: \ell \in h} A_{h \ell}} .
$$

It is key to note here that swapping the summations is allowed given that the VoronoiDelaunay decomposition of the volumes determines a tiling of the manifold without overlap. This will generally be true for arbitrary triangulations with circumcentric duals as long as volume orientation is also carried over in the calculation. Again, dividing by the integral volume, we get an explicit expression for the Ric weighting on an edge of the simplicial lattice;

$$
R_{\ell}=d(d-1) \frac{\left\langle\epsilon_{h}\right\rangle_{\ell}}{\left\langle A_{h^{*}}\right\rangle_{\ell}} \quad(\text { for } d>2)
$$




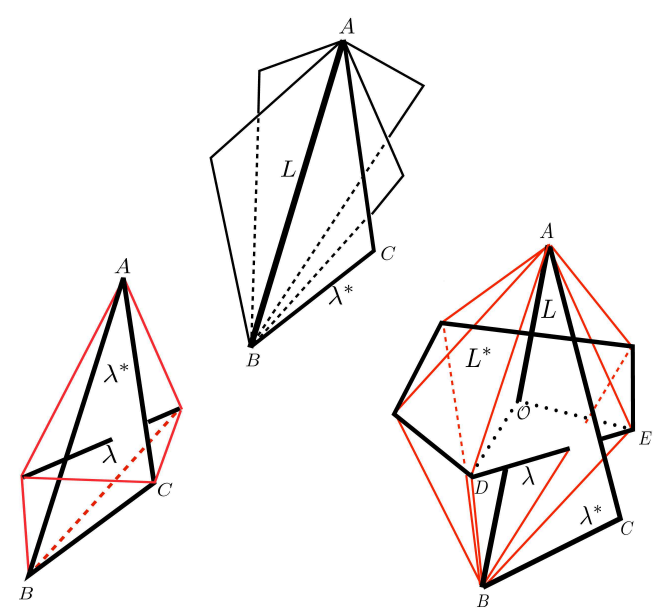

Figure 2. Volumes for the Ric on a Simplicial Hinge: Here we use the case of $d=3$ as a concrete example of the construction of the simplicial Ric from the dual edge-based Ric. (Top) A simplicial edge $\ell$ is shown with all triangles $t=\lambda^{*}$ hinging on $\ell$. The notation of $t=\lambda^{*}$ indicates that to each triangle containing $\ell$, there is an edge $\lambda$ of the dual lattice orthogonal and dual to $t$. (Bottom-left) The 3 -volume for a given $\lambda$ is depicted here. In general, only a portion of this volume will overlap with the 3 -volume associated with $\ell$. To construct the Ric for $\lambda$, the integral volumes used must coincide, so we take the restriction of $\Delta V_{\lambda}$ to $\ell, \Delta V_{\lambda \ell}$. (Bottom-right) The 3 -volume $\Delta V_{\ell}$ is shown and we indicate the part of $\Delta V_{\ell}$ corresponding to $\Delta V_{\lambda \ell}$ as the volume spanned by the vertexes $[A B D E \mathcal{O}]$. Since $\ell^{*}=h^{*}$, summing over all $\lambda$ contained in $h^{*}$ carries us around the loop orthogonal to $\ell$. In the restriction of $\Delta V_{\lambda}$ to $\ell$, the only contribution with non-trivial restricted volume is $h^{*}=\ell^{*}$ for the given $\ell$. Hence, substituting the expression for $R_{\lambda}$ into Eq. 13 and summing over all $\lambda \in \ell^{*}=h^{*}$, we obtain the Regge curvature on an edge/hinge in $d=3$. Hence we have $R_{\ell} \Delta V_{\ell}=R_{h} \Delta V_{h}$ as expected.

For the special case of $d=3$, the Riemann tensor is proportional to the Ric, i.e. all curvature content is encoded directly in the Ricci tensor. In Figure 2 we look at the Ric on a simplicial edge in $d=3$ and illustrate the volumes associated with the construction.

We now turn to the special case of $d=2$. The duality between $\lambda$ and $\ell$ is such that

$$
\begin{aligned}
R_{\ell} \Delta V_{\ell} & =\sum_{\lambda \in \ell^{*}} R_{\lambda} \Delta V_{\lambda \ell} \\
& =\sum_{\lambda} R_{\lambda} \Delta V_{\lambda} \delta_{\lambda, \ell^{*}}=R_{\lambda} \Delta V_{\lambda} .
\end{aligned}
$$

Again, the duality can be used to show $R_{\ell}=R_{\lambda}$. Using the expression for $R_{\lambda}=\overline{\left\langle R_{h=v}\right\rangle_{\lambda}}$ we have

$$
\begin{aligned}
R_{\ell} & =\frac{\sum_{h=v \ni \ell} R_{h} A_{h \ell}^{*}}{\sum_{h=v \ni \ell} A_{h \ell}^{*}} \\
& =\frac{\sum_{h=v \ni \ell} R_{h} \frac{1}{4} \ell \times \ell^{*}}{\frac{1}{2} \ell \times \ell^{*}} \\
& =\left.\bar{R}_{h}\right|_{\ell}
\end{aligned}
$$


where $\left.\bar{R}_{h}\right|_{\ell}$ is the arithmetic average of the curvature evaluated at the endpoints of $\ell$. We have used the normalization of $\operatorname{Vol}(h=v)=1$ in the first equality and the relation $A_{h \ell}=\frac{1}{4} \ell \times \ell^{*}=A_{h^{\prime} \ell}$ for both endpoints (hinges), $h$ and $h^{\prime}$, on $\ell$ in the second equality. This is a discrete expression showing explicitly that the Ricci one-form is determined solely by the scalar curvature (on vertexes) in 2-dimensions.

\subsection{Derivation of the Ric from the $R C$ Action Principle}

These expressions can also be derived from the Regge action principle in a similar way to the authors' previous construction of the scalar curvature invariant in RC [26]. Since the curvature is locally proportional to the sectional curvature, we obtain a simple relation between the action using the curvature two-form and the canonical Einstein-Hilbert action;

$$
I=\frac{1}{\kappa} \sum_{h}\left(\mathbf{R}, \mathbf{h}^{*}\right)=\frac{1}{\kappa} \sum_{h} d(d-1) K_{h} \Delta V_{h}=\frac{2}{\kappa} \sum_{h} \epsilon_{h} A_{h}=I_{\text {Regge }}
$$

where $\mathbf{h}^{*}$ is the two-form for the dual loop to a hinge and $K_{h}=\frac{\epsilon_{h}}{A_{h}^{*}}$ is the sectional curvature. The factor of $d(d-1)$ comes about from contracting the curvature twoform with the dual polygon two-form which gives equal contributions from all non-zero components. Using duality, we can also change the first expression to a hinge-based, instead of a dual polygon, expression;

$$
I=\frac{1}{\kappa} \sum_{h}(\star \mathbf{R}, \mathbf{h}) .
$$

Now tracing over directions orthogonal to edges and summing over the edges we get

$$
I=\frac{1}{\kappa} \sum_{h} \sum_{\ell \in h}(d-1)\left(R_{h \ell}, \ell\right)
$$

where $R_{h \ell}$ is the Ric on hinge $h$ directed along $\ell$. Contracting the Ric with its associate one-form gives an additional factor of $d$ such that we obtain

$$
I=\frac{1}{\kappa} \sum_{h} \sum_{\ell \in h} d(d-1) K_{h} \Delta V_{\ell h} .
$$

To get the action in terms of the Ricci one-form we decompose the integral measures and rearrange the summations;

$$
\begin{aligned}
I & =\sum_{h} d(d-1) \frac{\epsilon_{h}}{A_{h}^{*}} \underbrace{\frac{1}{\left(\begin{array}{l}
d \\
2
\end{array}\right)} \sum_{\ell \in h} A_{h}^{*} A_{h \ell}}_{\Delta V_{\ell h}} \\
& =\sum_{h} \sum_{\ell \in h} d(d-1) \frac{\epsilon_{h}}{A_{h}^{*}} \frac{1}{\left(\begin{array}{l}
d \\
2
\end{array}\right)} A_{h}^{*} A_{h \ell} \\
& =\sum_{\ell} \sum_{h: \ell \in h} d(d-1) \frac{1}{\left(\begin{array}{l}
d \\
2
\end{array}\right)} \frac{\epsilon_{h}}{A_{h}^{*}} A_{h}^{*} A_{h \ell} \\
& =\sum_{\ell} R_{\ell} \Delta V_{\ell} .
\end{aligned}
$$


Using the equality of the individual terms in the sum over edges, we get an expression for the curvature on an edge of the simplicial lattice;

$$
R_{\ell} \Delta V_{\ell}=\sum_{h: \ell \in h} d(d-1) \frac{1}{\left(\begin{array}{c}
d \\
2
\end{array}\right)} \frac{\epsilon_{h}}{A_{h}^{*}} A_{h}^{*} A_{h \ell}
$$

We have absorbed the combinatoric factor of $d(d-1)$ into the definition of $R_{\ell}$ as we will do in general. This helps keep in mind that the expression for $R_{\ell}$ is a scalar weight on the edge element. Formally, these scalar weights are part of a integrated quantity and are not necessarily assigned to a point on the lattice, but rather across the domain of integration associated with the given element. Hence, the curvature forms used in RC are to be understood as $R_{h} \Delta V_{h}, R_{\ell} \Delta V_{\ell}$, and $R_{v} \Delta V_{v}$ for the Riemann, Ricci and scalar curvature, respectively.

We can raise the simplicial Ricci one-form to obtain the dual Ricci one-form. To do so, we first restrict the integrative domain to the volume closest to the dual edge;

$$
R_{\ell} \Delta V_{\ell \lambda}=\sum_{h: \ell \in h} \frac{d(d-1)}{\left(\begin{array}{l}
d \\
2
\end{array}\right)} \frac{\epsilon_{h}}{A_{h}^{*}} A_{h \lambda}^{*} A_{h \ell} .
$$

We define the raising (lowering) operation applied to the simplicial Ric by summing over all integrated $R_{\ell}$ for which $\lambda \in \ell^{*}$. The restriction of the domain above is necessary to ensure that lowering (raising) this expression gives a quantity integrated over the appropriate $d$-volume. Doing so we obtain

$$
\begin{aligned}
R_{\lambda} \Delta V_{\lambda} & =\sum_{\ell: \lambda \in \ell^{*}} R_{\ell} \Delta V_{\ell \lambda} \\
& =\sum_{\ell: l \in \ell^{*}} \frac{d(d-1)}{\left(\begin{array}{l}
d \\
2
\end{array}\right)} \sum_{h: \ell \in h} \frac{\epsilon_{h}}{A_{h}^{*}} A_{h \lambda}^{*} A_{h \ell} \\
& =\sum_{h: \lambda \in h^{*}} \frac{d(d-1)}{\left(\begin{array}{l}
d \\
2
\end{array}\right)} \sum_{\ell \in h} \frac{\epsilon_{h}}{A_{h}^{*}} A_{h \lambda}^{*} A_{h \ell} \\
& =\sum_{h: \lambda \in h^{*}} \frac{d(d-1)}{\left(\begin{array}{l}
d \\
2
\end{array}\right)} \frac{\epsilon_{h}}{A_{h}^{*}} A_{h \lambda}^{*} A_{h} \\
& =\sum_{h: \lambda \in h^{*}} 2 \frac{\epsilon_{h}}{A_{h}^{*}} A_{h \lambda}^{*} A_{h} .
\end{aligned}
$$

Comparing with Eq. (9) shows exact agreement. The independence of the local and global derivations shown here are indicative of the decomposition of the lattice into elements with compact support. Therefore, the global derivation in terms of the action becomes just an additional sum over the local terms defined over the domains of compact, local support. This highlights the reason that $\mathrm{RC}$ as a weak variational principle reduces to locally simple characterizations of the manifold geometry.

\section{The Canonical Einstein Tensor}

In previous work, the Cartan moment-of-rotation trivector view was used to derive the embedding of the Einstein tensor in $\mathrm{RC}$ [25, 28]. Here we present an alternative 
derivation using the more familiar definition of the Einstein tensor;

$$
G_{\mu \nu}=R_{\mu \nu}-\frac{1}{2} g_{\mu \nu} R
$$

which can be rewritten as an Einstein one-form

$$
G_{a} \equiv G_{\mu \nu} e_{a}^{\nu}=R_{a}-\frac{1}{2} e_{a} R
$$

Using the simplicial Ric and the previously derived scalar curvature [26], we have all the necessary tools to provide a direct reconstruction of the Einstein tensor on an edge.

The isomorphism between forms on the dual and forms on the simplicial lattice allows us the freedom to define curvature forms on either lattice. However, we should start off on a sound geometric footing by following the projection of the continuum object onto the lattice structure. Eq. (26) identifies the quantitative construction of the Einstein tensor, but does not indicate the geometric character of the Einstein oneform. However, it is known that the Einstein tensor is the double-dual of the Riemann curvature tensor [29];

$$
G_{i}^{j}=\left({ }^{*} R^{*}\right)_{i m}{ }^{j m}=\frac{1}{4} \epsilon_{m n i l} R_{a b}^{m n} \epsilon^{a b l j} .
$$

The Hodge duals transform the two-form components on the dual lattice to forms on the simplicial lattice. The trace over the second and third indices reduce the two-form to a one-form. Hence, the Einstein tensor is a one-form on edges of the simplicial lattice. Equivalently, in 4-d the Einstein one-form is the dual of the moment of rotation 3-form projected on the 3 -volume dual to an edge [25, 28]. We take the natural embedding for the Einstein one-form in RC to be on the simplicial 1-skeleton. One could easily construct a dual lattice Einstein one-form, though we see no particular benefit.

We must also be careful in how we introduce the vertex-based scalar curvature, $R_{v}$, in the edge-based representation. This is most directly accomplished by projecting the integrated scalar curvature at a vertex onto the $d$-volume associated with an edge;

$$
e_{a} R \longrightarrow R_{v} \Delta V_{v \ell}
$$

This contributes non-trivially only when the vertex $v$ is a vertex of $\ell$. Moreover, since the scalar curvature is decomposed into volumes associated with the hinges meeting at $v$, this projection introduces a Kronecker delta into each term. This results from projecting the vertex-based volume associated with a hinge $\Delta V_{h v}$ onto a given edge. Hence, only those hinges meeting at $\ell$ contribute to the edge-restricted scalar curvature;

$$
\begin{aligned}
R_{v} \Delta V_{v \ell} & =d(d-1) \sum_{h: v, \ell \in h} \frac{\epsilon_{h}}{A_{h}^{*}} \frac{1}{\left(\begin{array}{c}
d \\
2
\end{array}\right)} A_{h \ell v} A_{h}^{*} \\
& =2 \sum_{h: v, \ell \in h} \epsilon_{h} A_{h \ell v}
\end{aligned}
$$

where $A_{h \ell v}$ is the area of the hinge $h$ restricted to both the edge $\ell$ and the vertex $v$ - both $\ell$ and $v$ are assumed to be on $h$ otherwise $A_{h \ell v}=0$. 
Using this representation of the scalar curvature and the simplicial Ricci one-form defined above, we are in position to explicitly define the canonical form of the Einstein tensor;

$$
\begin{aligned}
G_{\ell} \Delta V_{\ell} & =R_{\ell} \Delta V_{\ell}-\frac{1}{2} \sum_{v \in \ell} R_{v} \Delta V_{v \ell} \\
& =2 \sum_{h: \ell \in h}\left(\epsilon_{h} A_{h \ell}\right)-\sum_{v \in \ell} \sum_{h: \ell \in h} \epsilon_{h} A_{h \ell v} \\
& =2 \sum_{h: \ell \in h}\left(\epsilon_{h} A_{h \ell}\right)-\sum_{h: \ell \in h} \epsilon_{h} A_{h \ell} \\
& =\sum_{h: \ell \in h} \epsilon_{h} A_{h \ell} .
\end{aligned}
$$

In $d=4$ this becomes

$$
\begin{aligned}
G_{\ell} \underbrace{\frac{1}{4} \ell \cdot \ell^{*}}_{\Delta V_{\ell}} & =\sum_{h: \ell \in h} \epsilon_{h} A_{h \ell} \\
& =\sum_{h: \ell \in h} \epsilon_{h} \underbrace{\frac{1}{2} \ell \cdot \frac{1}{2} \ell \cot \left(\theta_{h \ell}\right)}_{A_{h \ell v}} \\
G_{\ell} \ell^{*} & =\sum_{h: \ell \in h} \epsilon_{h} \ell \cot \left(\theta_{h \ell}\right)
\end{aligned}
$$

where $\theta_{h \ell}$ is the angle on the hinge $h$ opposite $\ell$. Staying in $d=4$ we can check this result with the result obtained from varying the Regge action. In the continuum the integrated Einstein tensor is obtained from the variational principle;

$$
\int \sqrt{-g} G_{\alpha \beta} d^{4} x=\kappa \frac{\delta I_{\text {geom }}}{\delta g^{\alpha \beta}}
$$

where $\kappa=16 \pi G c^{-4}$ and $I_{\text {geom }}$ is the Einstein-Hilbert action.

In $\mathrm{RC}$, with action given by $\frac{2}{\kappa} \sum_{h} \epsilon_{h} A_{h}$, this becomes

$$
G_{\ell} \ell^{*}=\kappa \frac{\delta I_{\text {Regge }}}{\delta \ell}
$$

Regge showed that the variation of the deficit angle $\epsilon_{h}$ in the Regge action does not contribute to the final equations of motion. Only variation of the hinge volume contributes. Using this result we obtain the standard Regge equations for an edge;

$$
\begin{aligned}
\frac{\delta I_{\text {Regge }}}{\delta \ell} & =2 \frac{1}{\kappa} \sum_{h: \ell \in h} \epsilon_{h} \frac{1}{2} \ell \cot \left(\theta_{\ell h}\right) \\
& =\frac{1}{\kappa} \sum_{h: \ell \in h} \epsilon_{h} \ell \cot \left(\theta_{\ell h}\right) .
\end{aligned}
$$

The integrated Einstein tensor from the variational principle is thus found to match the result obtained from the Regge version of the canonical Einstein tensor definition;

$$
G_{\ell \ell} \ell^{*}=\sum_{h: \ell \in h} \epsilon_{h} \ell \cot \left(\theta_{\ell h}\right) .
$$


This agrees with the results from the moment of rotation three-form derivations [25, 28]. The factor of 2 that explicitly appears in Eq. (36) that cancels the $\frac{1}{2}$ factor in the moment arm is due to combinatoric factors coming from the symmetry in the moment of rotation, i.e.

$$
\mathbf{d} \mathcal{P} \wedge \mathbf{R}=\mathbf{R} \wedge \mathbf{d} \mathcal{P}
$$

In particular, the integrated moment of rotation assigned to an edge is not dependent on the ordering of the wedge product of the moment arm with the curvature and gives rise to this numerical factor.

It is particularly instructive to confirm this result by way of Eq. (28). Since the first dual acts on the space of values, we only need note that one component survives while the second component of the bivector contributes to the trace. Acting on the twoform components is the fundamental volume form, $\epsilon^{a b l j}$, which acts as a given 4-volume. Choosing a given component of $G_{i}$ is akin to choosing an edge $\ell$ on the simplicial lattice. Since $R_{h}=R_{h^{*}}$ takes non-zero components only in the directions orthogonal to hinges, the trace is the sum over directions orthogonal to $\ell$ and $h^{*}$;

$$
\begin{aligned}
\left(G^{j}, \ell^{j}\right) & =\frac{1}{2} \sum_{h: \ell \in h} R_{h^{*}} \Delta V_{h^{*} \ell} \\
& =\frac{1}{2} \sum_{h: \ell \in h} d(d-1) \frac{\epsilon_{h}}{A_{h^{*}}} \frac{1}{\left(\begin{array}{l}
d \\
2
\end{array}\right)} A_{h \ell} A_{h^{*}} \\
G_{\ell} \Delta V_{\ell} & =\frac{1}{4} \sum_{h: \ell \in h} \epsilon_{h} \ell^{2} \cot \left(\theta_{\ell h}\right)
\end{aligned}
$$

where we have used $A_{h \ell}=\frac{1}{2} \ell^{2} \cot \left(\theta_{\ell h}\right)$. Doing the usual trick of decomposing the volume on the LHS and dividing by $\ell$, we have

$$
G_{\ell} \ell^{*}=\sum_{h: \ell \in h} \epsilon_{h} \ell \cot \left(\theta_{\ell h}\right)
$$

as before. In general, the Einstein tensor in arbitrary dimension is given by;

$$
G_{\ell} \ell^{*}=\frac{d}{\ell} \sum_{h} \epsilon_{h} A_{h \ell}
$$

in agreement with Eq. (31). We thus have multiple methodologies for deriving the Einstein tensor, and we have shown that the Einstein tensor is the sum of restricted areas of hinges times their associated deficit angles.

\section{Conclusion}

We have presented here the first geometric discretization of the Ric in RC in arbitrary dimension. The tracing of the Riemann tensor over loops of parallel transport produces a one-form in the dual lattice. Moreover, we are able to use the isomorphism between forms on the dual with forms on the simplicial lattice to construct a simplicial counterpart to the dual lattice Ricci one-form. Both formulations provide explicit meaning to the simplicial analog of the trace of the Riemann tensor as an edge-based 
"weighted average" of curvature. In the dual representation the Ric is a volumeweighted average while in the simplicial representation it becomes a ratio of areaweighted averages.

The Ric defined as one-form in the simplicial or dual lattices is one step towards accurately embedding the machinery of RF into the piecewise-flat discretization of RC. By representing the Ric, and eventually $\mathrm{RF}$, in the $\mathrm{RC}$ framework, we expect to be able to use RF on geometries of arbitrary topology in arbitrary dimension. In particular, the 3-dimensional Ric carries the full information about the curvature of the manifold and can be used for manifold comparison using techniques developed by Perelman [2, 3, 4, 30]. Ongoing future work will develop the RF equations and apply them to discrete manifolds in higher dimension.

The definition of a Ric in arbitrary dimension has further allowed us to provide a third and independent derivation of the Einstein tensor in RC. By using our simplicial

Ricci one-form and the recent definition of the vertex-based scalar curvature, we are able to write an explicit expression for the trace-reversed Ric in terms of restricted volumes in the simplicial lattice. This shows further the utility of the inherent Voronoi-Delaunay duality and the associated hybrid cells as natural volumes in RC.

\section{Acknowledgments}

We would like to thank Shing-Tung Yau and Xianfeng $\mathrm{Gu}$ for stimulating our interest in this topic and pointing out useful references. JRM would like to acknowledge partial support from the SFB/TR7 "Gravitational Wave Astronomy" grant funded by the German Research Foundation (DFG) and is currently supported through a National Research Council Research Associateship Award at AFRL Information Directorate. WAM acknowledges partial support from the Information Directorate at Air Force Research Laboratory. PMA wishes to acknowledge the support of the Air Force Office of Scientific Research (AFOSR) for this work. Any opinions, findings, and conclusions or recommendations expressed in this material are those of the authors and do not necessarily reflect the views of AFRL.

\section{Appendix A. Integral Volumes in Regge Calculus}

The canonical volumes of $\mathrm{RC}$ are the simplicial blocks of the lattice. These domains define the locally flat subspaces of the geometry. The simplicial blocks also supply the lattice with an intrinsice definition of local tangent spaces on which we explicitly define vectors, tensors, and differential forms. It is useful to decompose these simplicial domains to fit with the character of the geometric objects we construct. Since all embeddings of geometric variables are essentially integrated quantities, as opposed to the point-based representation in the continuum, we wish the integral volumes to reflect the nature of the object itself. Here we provide a short review of the methods for constructing integral volumes used in this manuscript. 
We begin by defining the simplicial volume via the inner-product of forms. The volume of a simplicial cell is given by the inner product of the simplicial $d$-form with itself;

$$
\begin{aligned}
\left(s^{(d)}, s^{(d)}\right) & =\int s^{(d)} \wedge * s^{(d)} \\
& =\frac{1}{\left(\begin{array}{l}
d \\
d
\end{array}\right)}\left|s^{(d)}\right| \cdot\left|* s^{(d)}\right|=\left|s^{(d)}\right|
\end{aligned}
$$

where we use the usual notation, $|\cdot|$, to indicate the norm. Since $* s^{(d)}$ is a vertex of the dual lattice, i.e. the circumcenter of $s^{(d)}$, it contributes only a scalar constant to the integral. To ensure that the integral yields the appropriate $d$-volume, we choose assign to any vertex a volume with unit normalization. Likewise, a polytope $\sigma^{(d)}$ dual to a vertex $v$ in the simplicial lattice is given by

$$
\left(* \sigma^{(d)}, * \sigma^{(d)}\right)=\frac{1}{\left(\begin{array}{l}
d \\
0
\end{array}\right)}\left|* \sigma^{(d)}\right| \cdot\left|\sigma^{(d)}\right|=\left|\sigma^{(d)}\right|
$$

where again we have $\left|* \sigma^{(d)}\right|=|v|=1$. Explicitly, this volume is constructed by building local domains interior to each simplex in the star of the vertex $v$ dual to $\sigma^{(d)}$. Using the Voronoi construction, this volume localized on a simplex is the set points in polysd closest to $v$ than any other vertex in the simplex. This portion of the simplex will be called the restriction of the simplex to $v, \Delta V_{s^{(d)}} v=\left|s^{(d)}\right|_{v}$. Summing over each simplex in the star of $v, \operatorname{St}(v)$, gives the complete dual volume.

$$
|* v|=\sum_{s^{(d)} \in \operatorname{St}(v)}\left|s^{(d)}\right|_{v} .
$$

We can construct arbitrary volumes that are hybrid Delaunay-Voronoi cells through inner-products of the simplicial (dual) $r$-forms with themselves;

$$
\begin{aligned}
\left(s^{(r)}, s^{(r)}\right) & =\int s^{(r)} \wedge * s^{(r)} \\
& =\frac{1}{\left(\begin{array}{c}
d \\
r
\end{array}\right)}\left|s^{(r)}\right||* s r| .
\end{aligned}
$$

The factorization given by the last equatlity is a direct result of the inherent orthogonality between the Voronoi and Delaunay lattices. This canonical factorization is one of many factorizations. One may also decompose the volume associated with a given simplicial or dual element into volumes determined by $m$-forms $(m<r)$ contained in a given $s^{(r)}$ or $n$-forms $(n>r)$ in $\operatorname{St}\left(s^{(r)}\right)$;

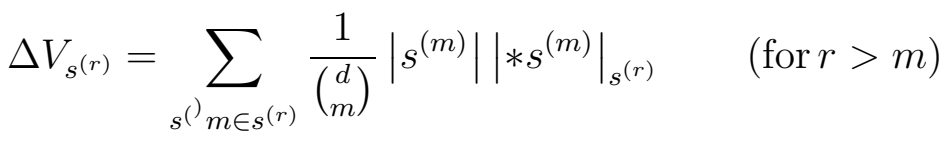

$$
\begin{aligned}
& \Delta V_{s^{(r)}}=\sum_{s^{(n)} \in \operatorname{St}\left(s^{(r)}\right)} \frac{1}{\left(\begin{array}{l}
d \\
n
\end{array}\right)}\left|s^{(n)}\right|_{s^{(r)}}\left|* s^{(n)}\right| \quad(\text { for } r<n) .
\end{aligned}
$$

Here, the Voronoi-Delaunay duality is again particularly useful as it allows us to construct the restricted volume via restriction of only a subspace of a given volume. 
The restriction is applied to the subspace such that the restriction makes sense, i.e. the restriction of $s^{(m)}$ to $s^{(r)}(r>m)$ trivially yields the norm $\left|s^{(m)}\right|$. Such restrictions are explicitly used in the definition of the vertex-based scalar curvature which require vertex $d$-volumes to be decomposed using the vertex-restriction of the hinge area [26]

\section{Appendix B. Operations on Discrete Forms}

In the lattice we endow the geometry with two distinct spaces of differential forms, (1) the simplicial skeleton as the representation of the homology and (2) the dual skeleton as the representation of the cohomology. The representation of differential forms on a simplicial complex is based on the ideas of Whitney [18] and has been used in computational electromagnetism [19, 20, 21] and computational geometry [12, 13]. The purpose of such a representation is to not just discretize tensor and differential form fields by representing their components point-wise on some discrete set of points, but to embed the full geometric character of a field in the discretization. In this way, one hopes to preserve the general geometric properties and symmetries of the field in the discretization. In this appendix we review some useful isomorphisms between the spaces of forms in the simplicial and dual lattices.

The first and most straightforward isomorphism is the Hodge dual. The Hodge dual maps an element of $\Lambda^{(r)}\left(\Lambda^{*(r)}\right)$ to $\Lambda^{*(d-r)}\left(\Lambda^{(d-r)}\right)$. This is defined by mapping the scalar weighting to a given simplicial (dual) element of the skeleton to its geometric dual, i.e.

$$
\alpha_{s}(r) \longrightarrow \alpha_{* s}(r)
$$

This is done via formal mapping [23]

$$
\frac{1}{\mid s^{(r) \mid}}\left\langle\alpha, s^{(r)}\right\rangle=\frac{1}{\left|* s^{(r)}\right|}\left\langle * \alpha, * s^{(r)}\right\rangle
$$

where $\langle\alpha, \Omega\rangle=\int_{\Omega} \alpha$. Since differential forms in RC are represented as scalar weights on elements of the lattice, this isomorphism is a simple mapping of the weight from an element on one lattice to its dual element.

We also can construct the raising (lowering) operations in the lattice. In the continuum, this operation is carried out via the metric or its inverse applied to components of the form. In the lattice, we must construct a way of identifing a scalar weighting to an $r$-form of the simplicial (dual) lattice using the weights of the $r$-forms in the dual (simplicial) lattice. We define the isomorphism taking dual $r$-forms to a simplicial $r$-form as

$$
\alpha_{s^{(r)}} \Delta V_{s^{(r)}}=\left\{\begin{array}{cl}
\sum_{\sigma^{()} r: s^{(r)} \in * \sigma^{()} r} \alpha_{\sigma^{(r)}} \Delta V_{\sigma^{(r)} s^{(r)}}, & \text { if } 2 r \leq d \\
\sum_{\sigma^{(r)}: * \sigma^{(r)} \in s^{(r)}} \alpha_{\sigma^{(r)}} \Delta V_{\sigma^{(r)} s^{(r)}}, & \text { if } 2 r>d
\end{array}\right.
$$


Using the orthogonal decomposition and restriction of volumes defined in Appendix Appendix A, the volumes on the RHS are given by

$$
\Delta V_{\sigma^{(r)} s^{(r)}}= \begin{cases}\frac{1}{\left(\begin{array}{c}
d \\
r
\end{array}\right)}\left|\sigma^{(r)}\right|\left|* \sigma^{(r)}\right|_{s^{(r)}}, & \text { if } 2 r \leq d \\
\frac{1}{\left(\begin{array}{c}
d \\
r
\end{array}\right)}\left|* \sigma^{(r)}\right|\left|\sigma^{(r)}\right|_{s^{(r)}}, & \text { if } 2 r>d\end{cases}
$$

One can define a similar isomorphism from the simplicial lattice to the dual lattice by taking the sum over elements of the simplicial skeleton. It is important here that we incorporate the restriction of the integral domain into the defintion to ensure that if we apply the inverse isomorphism that we reobtain the initial $r$-form. This can be easily checked.

[1] R.S. Hamilton. Three-manifolds with positive Ricci curvature. J. Diff. Geom., 17:255-306, 1982.

[2] Grisha Perelman. The entropy formula for the Ricci flow and its geometric applications. preprint arXiv:0211159 [math.DG], 2002.

[3] Grisha Perelman. Ricci flow with surgery on three-manifolds. preprint arXiv:0303109 [math.DG], 2003.

[4] Grisha Perelman. Finite extinction time for the solutions to the Ricci flow on certain threemanifolds. preprint arXiv:0307245 [math.DG], 2003.

[5] Tullio Regge. General relativity without coordinates. Nuovo Cimento, 19:558-571, 1961.

[6] E. Woolgar. Some applications of Ricci flow in physics. Can. J. Phys., 86(4):645-651, 2008.

[7] Shing-Tung Yau. Private Communications.

[8] Joseph Samuel and Sutirtha Roy Chowdhury. Geometric flows and black hole entropy. Class. Quantum Grav., 24(11):F47, 2007.

[9] Sergey N. Solodukhin. Entanglement entropy and the ricci flow. Phys. Lett. B, 646(5-6):268 $274,2007$.

[10] M. Carfora and A. Marzuoli. Smoothing out spatially closed cosmologies. Phys. Rev. Lett., 53(25):2445-2448, Dec 1984.

[11] M. Carfora and T. Buchert. Ricci flow deformation of cosmological initial data sets. In Proceedings of the 14th International Conference on Waves and Stability in Continuous Media, pages 118128, Hackensack, NJ, 2008. World Scientific Publishing Co.

[12] Xianfeng Gu and Shing-Tung Yau. Computing conformal structures of surfaces. Comm. Info. Sys., 2:121-146, 2002.

[13] Xianfeng $\mathrm{Gu}$ and Shing-Tung Yau. Global conformal surface parameterization. In SGP '03: Proceedings of the 2003 Eurographics/ACM SIGGRAPH symposium on Geometry processing, pages 127-137, Aire-la-Ville, Switzerland, Switzerland, 2003. Eurographics Association.

[14] Matthew Headrick and Toby Wiseman. Ricci flow and black holes. Class. Quantum Grav., 23(23):6683, 2006.

[15] Matthew Headrick, Sam Kitchen, and Toby Wiseman. A new approach to static numerical relativity and its application to KaluzaKlein black holes. Class. Quantum Grav, 27(3):035002, 2010 .

[16] G. Holzegel, T. Schmelzer, and C. Warnick. Ricci flow of biaxial Bianchi IX metrics. preprint arXiv:0706.1694 [hep-th], 2007.

[17] David Garfinkle and James Isenberg. The modeling of degenerate neck pinch singularities in Ricci flow by Bryant solitons. J Math. Phys., 49(7):073505, 2008.

[18] H. Whitney. Geometric Integration Theory. Princeton University Press, Princeton, NJ, 1957.

[19] A. Bossavit. Differential forms and the computation of fields and forces in electromagnetism. Eur. J. Mech., B10:474-488, 1991. 
[20] A. Bossavit. Computational Electromagnetism: Variational Formulations, Complementarity, Edge Element. Academic Press, Chestnut Hill, MA, 1998.

[21] Doug N. Arnold, Richard S. Falk, and R. Winther. Finite element, exterior calculus, homological techniques, and applications. Acta Numerica, 15:1-155, 2006.

[22] A. Okabe, B. Boots, and K. Sugihara. Spatial Tessellations: Concepts and Application of Voronoi Diagrams. Wiley, New York, 1992.

[23] Mathieu Desbrun, Eva Kanso, and Yiying Tong. Discrete differential forms for computational modeling. In SIGGRAPH '06: ACM SIGGRAPH 2006 Courses, pages 39-54, New York, NY, 2006. ACM.

[24] Warner A. Miller. The Hilbert action in Regge calculus. Class. Quantum. Grav., 14:L199-L204, 1997.

[25] Warner A. Miller. Geometrodynamic content of the Regge equations as illuminated by the boundary of a boundary principle. Foundations of Physics, 16:143-169, 1986.

[26] Jonathan R. McDonald and Warner A. Miller. A geometric construction of the Riemann scalar curvature in Regge calculus. Class. Quantum. Grav., 25:196017, 2008.

[27] S. Kobayashi and K. Nomizu. Foundations of Differential Geometry, volume 1. Interscience Publishers, New York, 1963.

[28] Adrian P. Gentle, Arkady Kheyfets, Jonathan R. McDonald, and Warner A. Miller. A Kirchhofflike conservation law in Regge calculus. Class. Quantum Grav., 26:015005, 2009.

[29] John Lighton Synge. Relativity: The General Theory. North-Holland Publishing Co., Amsterdam, 1960.

[30] Karsten Grove and Peter Peterson, editors. Comparison Geometry. Cambridge Univ. Press, Cambridge, 1997. 